\title{
Influence of the refractive index of liquids in the speckle pattern of multimode fibers
}

\author{
Carlos Galindez, Mauro Lomer*, Luis Rodriguez-Cobo, and J.M. Lopez-Higuera \\ Photonics Engineering Group, University of Cantabria, Av los Castros S/N, 39005, Santander, Spain
}

\begin{abstract}
The distribution of the intensity of each speckle depends on the relative phases of modes in the multimode fiber, so they are extremely sensitive to external perturbations of the fiber. These perturbations can locally appear in the fiber without disrupting the entire optical assembly. If the refractive index of the outside medium of the fiber is changed, it can cause variations in the speckle pattern at the fiber output. Thus, by changing the refractive index of the outside medium the speckle pattern at the output fiber varies and its influence can be observed. In this paper we demonstrate the influence of the refractive index of liquids in the speckle pattern obtained by a multimode fiber. In order to obtain greater sensitivity of the experimental measurement, the fiber is bent in a U-shape and immersed in a liquid. The core and cladding are 240 microns and 250 microns, respectively. The intensity speckle field is then captured by a CCD camera in digital image format and processed by the computer with a Matlab program. The portion of fiber exposed to the disturbance of the liquid is located 2 meters before the exit of the fiber. The portion of the fiber in contact with the liquid is curved with a radius of $2 \mathrm{~mm}$.
\end{abstract}

Keywords: multimode fiber sensing, speckle pattern, liquid index.

\section{INTRODUCTION}

The speckle pattern produced by coherent light is often used in optical metrology, where the granular structure of light is obtained through an optical roughened surface [1]. In this case the speckle pattern depends on the properties of coherence of the incident field and the surface characteristics. Another method to obtain the speckle pattern consists to use multimode optical fibers. Indeed, when the light that comes out from a fiber is projected on a white screen, the speckle pattern generated by the different modes that propagate in the multimode fiber can be observed. Since the speckle pattern in the output fiber varies slowly with time, this pattern is sensitive to disturbances such as temperature, pressure or vibration in the fiber, which can be obtained by a suitable speckle pattern processing [1]. By taking advantage of this characteristic, various schemes and assemblies of sensors based on the speckle generated in multimode optical fibers have been proposed [2-5]. Those configurations have advantages over conventional systems due to their low cost, insensitive to electromagnetic interference, flexible, lightweight and easy installation. Spillman et al. demonstrated a method using vibration sensing changes in the spatial distribution of energy in the exit end of a multimode fiber [2]. The authors implemented two experimental setups, the first speckle pattern is spatially filtered and the second speckle pattern is projected onto CCD array, where intensity changes are observed and related to vibration. Yu et al., propose a multimode fiber-sensing system using photorefractive bulk crystal to construct a fiber-speckle hologram [3]. The experiment shows that the sensitivity can be of the order of $0.05 \mu \mathrm{m}$ or even higher if a higher-mode sensing fiber is used. Zhang et al., report the development of a optical fiber displacement/strain sensor using the intensity variation of laser speckle pattern, sensor can be embedded in concrete for the detection and measurement of crack opening displacements [4]. Regez et al., propose an optical fiber strain measurement sensing mechanism capable to measure extremely low strains, in the order of 0-25 microstrains, with a resolution close to 0.1 microstrain [5]. Recently, Wang et al, reported the application of speckle in fiber and chemical sensor [6]. The experimental setup behaved in a short singlemode fiber length between two multimode fibers with core diameters of 9 and 50 microns, respectively. The structure is immersed in a cell, where a chemical fluid passes through it. They experiment with mixtures of glycerin and water, and data clearly show a relations between the speckle pattern and the detected intensity. In this paper we demonstrate the influence of the refractive index of liquids in the speckle pattern obtained by a multimode fiber. In order to obtain greater sensitivity of the experimental measurement, the fiber is bent in a U-shape and immersed in liquid. The core and cladding of the fiber are 240 and 250 micrometers, respectively. The intensity speckle field is then captured by a CCD

\footnotetext{
*Email: lomerm@unican.es
}

Speckle 2012: V International Conference on Speckle Metrology, edited by

Ángel F. Doval, Cristina Trillo, J. Carlos López-Vázquez, Proc. of SPIE Vol. 8413, 84131Q

(C) 2012 SPIE - CCC code: 0277-786X/12/\$18 · doi: 10.1117/12.978219 
camera coupled to the output end of the fiber. The digital image format is processed by the computer with a Matlab program. Experimental results are presented and discussed, using salt and water solution.

\section{THEORY}

In a multimode step index fiber the direction of propagation is conditioned by the ratio of refractive index between the core and the cladding. For a beam that propagates in the core and that is incident to the core-cladding interface at an angle $\theta$, the total internal reflection condition is achieved when $\theta$ equals or exceeds $\theta_{c}$. $\theta_{c}$ is the critical angle

complementary given by $\theta_{c}=\cos ^{-1}\left(n_{2} / n_{1}\right)$, where $n_{1}$ and $n_{2}$ are the refractive indices of core and cladding, respectively. If all the bound rays are launched at the input end of the straight fiber, the angle $\theta$ varies from $\geq \theta_{c}$ to $90^{\circ}$. If the core of the fiber is sufficiently large, two sets of propagated modes may be considered: i) low order modes, these modes that propagate parallel to the fiber axis, ie., With $\theta \approx \pi$, and ii) high order modes, these modes are made at an angle to the fiber axis between $\pi \leq \theta \leq \theta_{c}$. The number of modes, $\mathrm{M}$, which supports an optical fiber break index is given by the expression [7], $M=V^{2} / 2$, where $V$ is called normalized frequency given by, $V=(2 \pi a / \lambda)\left[\left(n_{1}\right)^{2}-\left(n_{2}\right)^{2}\right]^{1 / 2}$, where $a$ is the core radius, $\lambda$ wavelength the laser. For a gradient index fiber, the number of modes is: $M \approx V^{2} / 4$. For example, 200000 modes will be obtained for a multimode fiber step-index, $n_{1}=1.492$ and $n_{2}=1.402$, and core radius $a=125 \mu \mathrm{m}$, and when a HeNe laser $(\lambda=0.6328 \mu \mathrm{m})$ is used. Due to the number of speckle dots present in a pattern is approximately equal to $M$, a speckle pattern adaptable to the needs of the measured variable can be obtained just by choosing a fiber with a suitable diameter and consequently by determining the size of the speckle dots. In a fiber straight, if all conditions for propagation are met, the modes excited in the input fiber should emerge at the tip of the fiber. But, due to manufacturing imperfections such as irregularities in the core-deck interface, microcracks, variations in core diameter, etc., the modes are coupled randomly among them. Therefore, the output will have a complex spatial distribution of modes that have been transformed in the course of propagation in the fiber. The effect of the ambient temperature surrounding the fiber may also cause variations in the distribution of spatial modes. Then, the slowly variations in time of the speckle pattern that were observed on the screen when the output beam is projected fiber, can be caused by one of these manufacturing defects. From the above details, we may say that the speckle pattern is extremely sensitive to any disturbances on the optical fiber. One factor to consider is the finite thickness of the deck, where several studies have shown its influence on the propagated modes [8]. Hence, optical fiber can be seen as a symmetric waveguide with two conditions. First, when it has the jacket and second, when it is in the air $(n=1)$ is like a three-waveguide means. Consequently, any change in the refractive index of the external medium affects the modes that are propagating, particularly those at higher modes. In a straight multimode fiber, once the modal balance is established [9], the measured optical power variations in the fiber end are almost constant. In order to increase the sensitivity of the fiber to environmental changes, one possibility would be to bend the fiber in addition to locating the influence of the change. Indeed, numerous studies have been devoted to show the influence of the bends in the path loss [10], and their applications in sensors [11]. To show the influence of bends in the propagation modes, we briefly comment some relevant points. Considering a fiber with a U-bend (Figure 1), the propagation conditions change in the bend region, since the critical angle now depends on the curvature radius, and these conditions will be different in the inner and outer interfaces of the bend region. This new angle $\phi$ is determined by the expression:

$$
\phi_{1}=\sin ^{-1}\left[\left(\frac{R \pm \rho}{R+a}\right) \frac{n_{2}}{n_{1}}\right] \quad \text { and, } \quad \phi_{2}=\sin ^{-1}\left[\frac{R \pm \rho}{R+a}\right]
$$

For the outer interface, the maximum value of $\phi_{1}$ corresponds to the high order modes, while the minimum $\phi_{2}$ corresponds to the low order modes. Here $R$ is the bending radius, $a$ is the fiber core radius, $\rho$ is the rays 's entrance height ( $\rho$ come from the axis of the fiber). The angle incident at the inner interface is also changed, but their influence will be lower in this calculation. From equation (1), we can determine the rays that are going out from the fiber and that can influence the calculus the bending losses of the fiber as function of $R$. For a given value of $R$, the effect of the outside medium can be analyzed by measuring the refractive index $n_{o}$. To develop a first order approximation the effect of the fiber cladding can ignored, because the cladding thickness is very small. Now, considering the SnelI's law (Fig. 1): $n_{1} \cdot \sin \phi=n_{2} \cdot \sin \phi_{1}{ }^{\prime}=n_{2} \sin \phi^{\prime} \approx n_{o} \cdot \sin \gamma$, where $n_{o}$ is the refractive index of surrounding medium and $\phi_{1}{ }^{\prime}, \phi^{\prime}$ and $\gamma$, are the angles between the normal and each side of the cladding interface and with the outside medium. 
Straight region

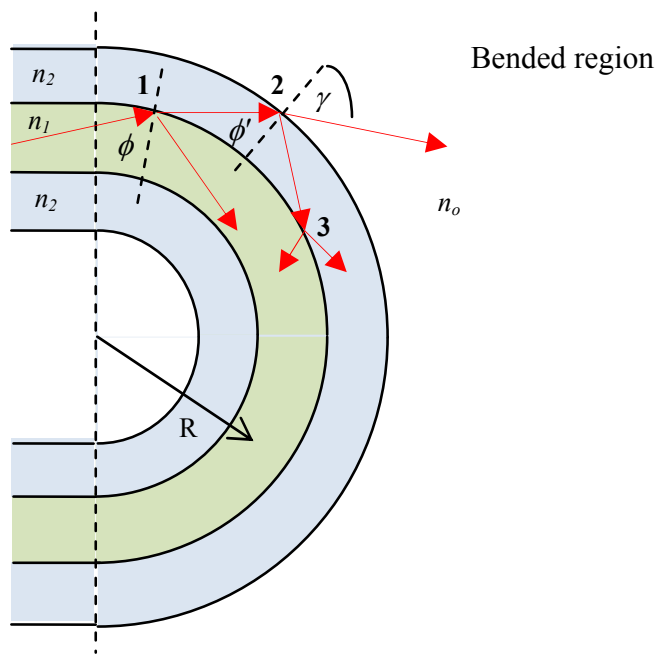

Figure 1. Structure of optical fiber bended of radius $R$ immersed in a medium of refractive index $n_{o}$.

To a first order of approximation one can ignore the effect of the fiber cladding, because the thickness to the cladding is very small, since according to SnelI's law (Fig. 1): $n_{1} \cdot \sin \phi=n_{2} \cdot \sin \phi_{1}{ }^{\prime}=n_{2} \sin \phi^{\prime} \approx n_{o} \cdot \sin \gamma$, where $n_{o}$ is the refractive index of surrounding medium and $\phi_{1}^{\prime}, \phi^{\prime}$ and $\gamma$, the angles to the normal on either side of the interface between cladding and surrounding medium. The critical angle, $\theta_{c}$, for ray at point 1 which is totally reflected and continuous to propagate in the fiber can be written as

$$
\theta_{c}=\arcsin \left[\frac{n_{o}}{n_{1}} \cdot \frac{\sin \phi^{\prime}}{\sin \phi}\right] \approx \arcsin \left[\frac{n_{o}}{n_{1}}\right]
$$

When $\phi<\theta_{c}$, most of the light energy is transmitted into the surrounding medium. This transmission is governed by Fresnel reflection coefficients. In equation (2), it can be clearly seen that the bent fiber is influenced by the refractive index of the external environment, so it implies that the system is sensitive to liquid media and their concentrations.

\section{SENSOR DESCRIPTION AND EXPERIMENTAL SETUP}

The experimental system made is shown in Fig. 2. The laser beam (HeNe, $\lambda=0.6328$ micrometer) is coupled into the multimode fiber. In our experiment, a Super Eska ${ }^{\mathrm{TM}}$ SK-10 multimode step-index POF is used. The refractive index of the core is 1,492 ; the core diameter is 240 micrometers and the numerical aperture is 0.5 . The head sensor is shown in Figure 3. It is made of an acrylate base that holds $20 \mathrm{~cm}$ of plastic optical fiber; the POF is bent with a curvature radius of $2 \mathrm{~mm}$. A total of $20 \mathrm{~m}$ of fiber is used, hence the cladding modes are stabilized and the use of scrambler is avoided. The curved part of the POF is immersed in a liquid sample to analyze the speckles pattern variation. The variation pattern is measured by using the setup depicted in Figure 3. An optical light coming from a HeNe laser is injected in the POF with the help of a micrometric positioning block; in the opposite extreme of the fiber a CCD (charge-coupled device) camera records the speckle pattern that is formed into the plastic fiber. The procedure to take the images is as follows: First, the camera is programmed to take pictures with a rate of 2 or 1 frames per second, later it starts to record. The first frames taken by the camera correspond to pictures of the speckles pattern of the sensor placed in the air, and then it is immersed in a liquid and taken again to the air. This procedure is repeated once more. Thus, the data is a sequence of speckles pattern images that register the conditions of the sensor head. 


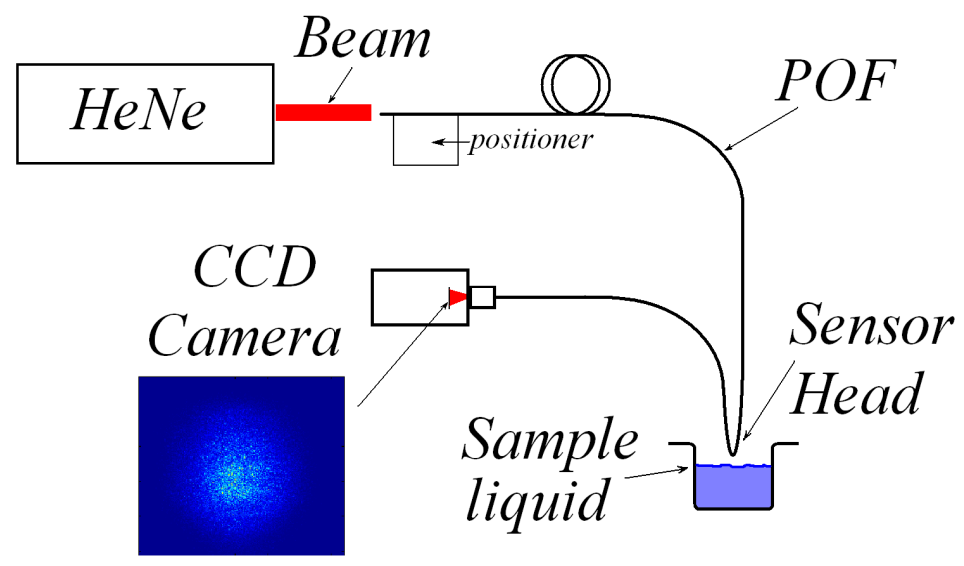

Figure 2. Schematics of the experimental setup in this study.

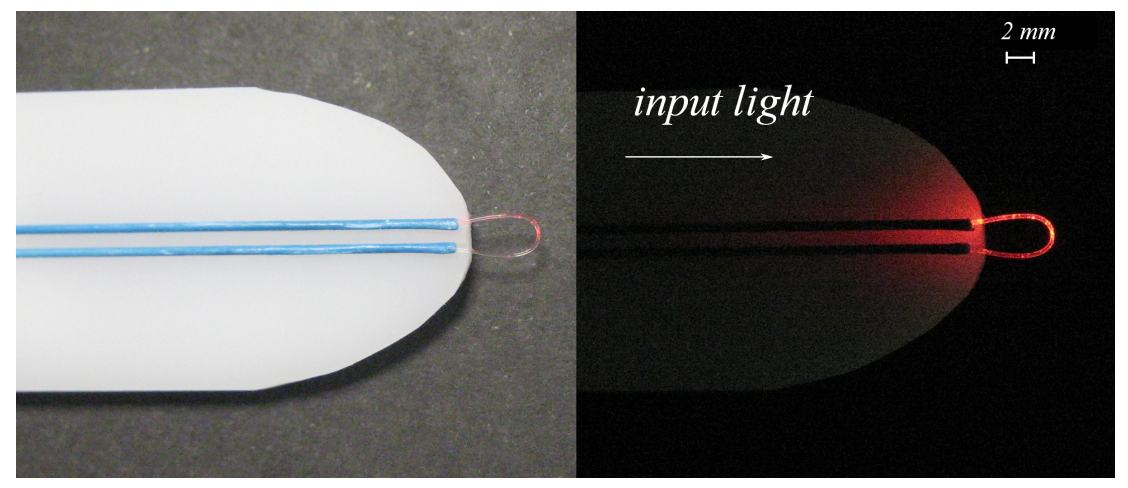

Figure 3. Sensor head.

\section{EXPERIMENTAL RESULTS AND DISCUSSION}

The speckles pattern varies each time the liquid covers the sensor head. The most visible variation is that one related to the intensity of the whole pattern captured by the CCD camera. The speckles pattern intensity decreases, respect to the one measured in the air, for liquids that surround the sensor head with higher refraction index than the air. This fact is shown in Figure 4. The three upper figures shown three frames captured by the CCD camera in three conditions. The figure named Frame 1 represents the speckles pattern for the sensor head in the air; the center figure (Frame 90) corresponds to an image taken when the sensor is immersed in water with salt, and the right upper figure shows the speckles pattern when it is taken back to air. From the first and third images, it can be easily deduced that they are brighter than the center image, which correspond to the sensor in the air. The procedure chosen for this work uses the idea of the Temporal History Speckles Pattern (THSP). From each frame is taken a section of 1x1024 pixels to form a new image with the frames captured in the experiment; in the case of the Figure 4 were captured 200 frames, then the new image is a matrix of 200x1024. This new image (lower image in Figure 4) shows information of the color scale given by the camera, which is proportional to the intensity of the speckles pattern in every condition of the sensor. Normalizing this parameter, the intensity losses respect to the conditions on the air is obtained. Hence, the intensity variations can be depicted and identified (Figure 5).

The same procedure above described can be used for obtaining the figures of intensity losses for water and index matching liquid. The results are depicted in Fig. 6. From those figures it can be observed that, the losses are lower for refraction index values closer to the air-index. 
In Figure 5 while it can be seen that there is loss difference between water and diluted with salt water by $20 \%$. This difference can be explained by the fact that the index of refraction of salt $(\mathrm{NaCl})$ in solid form and diluted in water is greater than the rate of water. Thus, when the fiber is immersed in salt water, the index of the medium outside the submerged fiber is greater than water, thus the losses will increase and the intensity detected at the tip of the fiber falls slightly. The loss difference between the two measures (water and salt water) is $0.6 \mathrm{~dB}$. Figure 6 shows the difference between the index matching liquid $\left(n_{o}=1.46\right)$ relative to air is greater than the difference when the sensor head is submerged in water $\left(n_{o}=1.33\right)$. These results demonstrate the influence of rate of liquid on the speckle pattern as well as the sensitivity of their concentration observed in very small variations of refractive index.

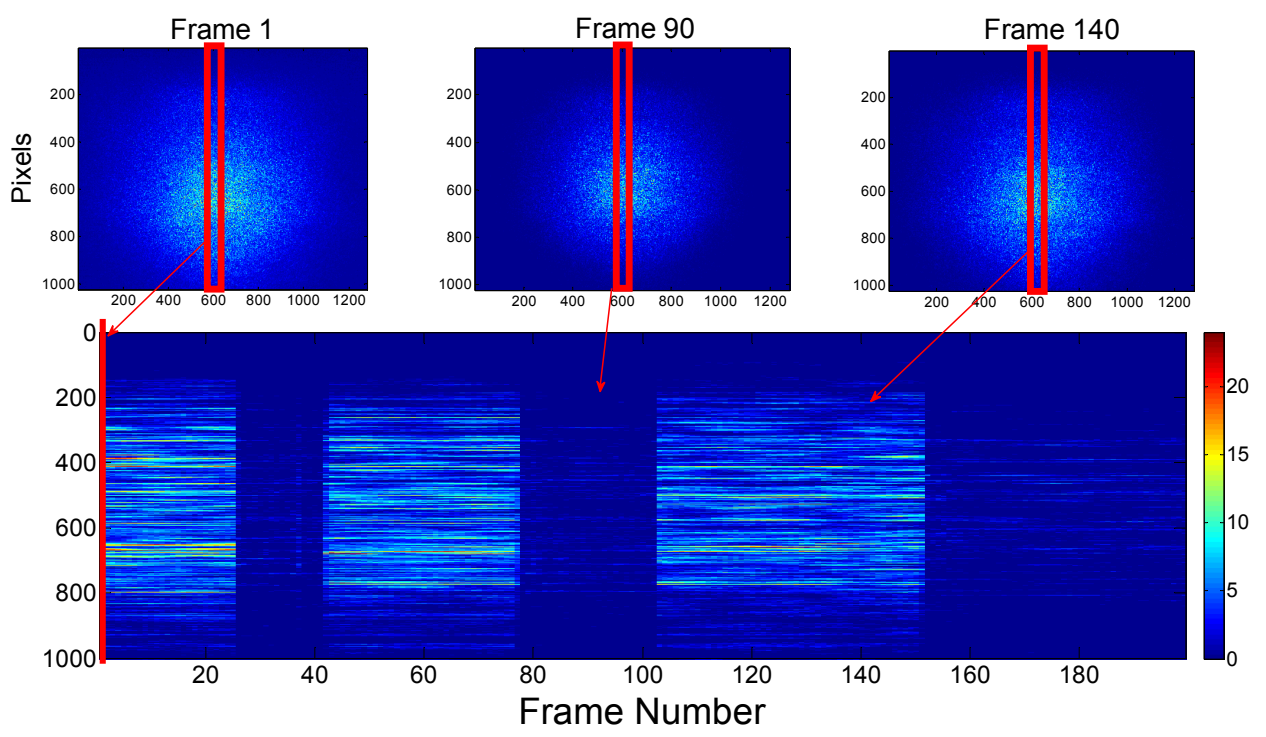

Figure 4. Speckles pattern captured when the head sensor is in the air, immersed in water with salt and taken back to air (Upper figures). Image obtained by using the THSP procedure (Lower figure).

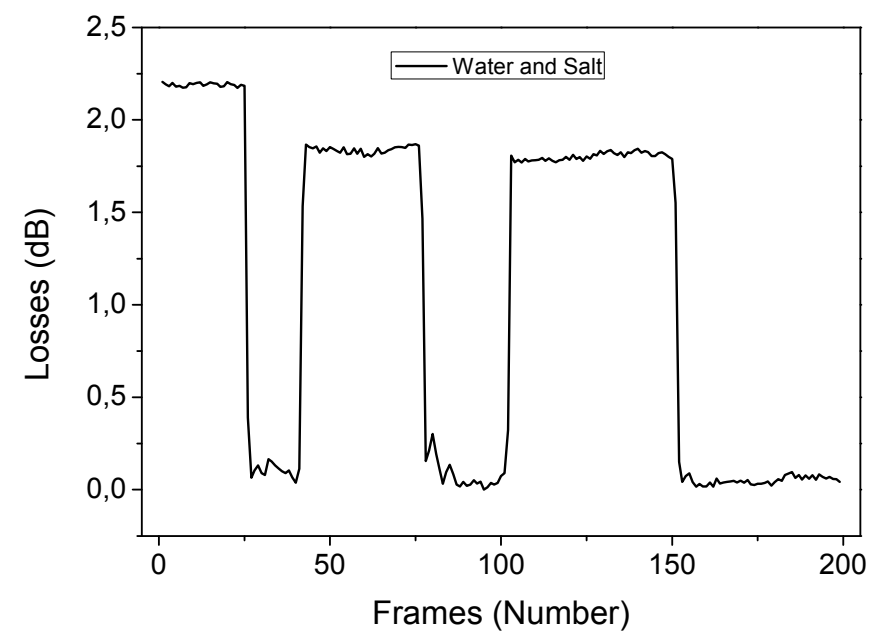

Figure 5. Normalized losses of a speckles pattern section (1x1024) calculated from the THSP matrix for Index matching liquid (left) and water (right). 


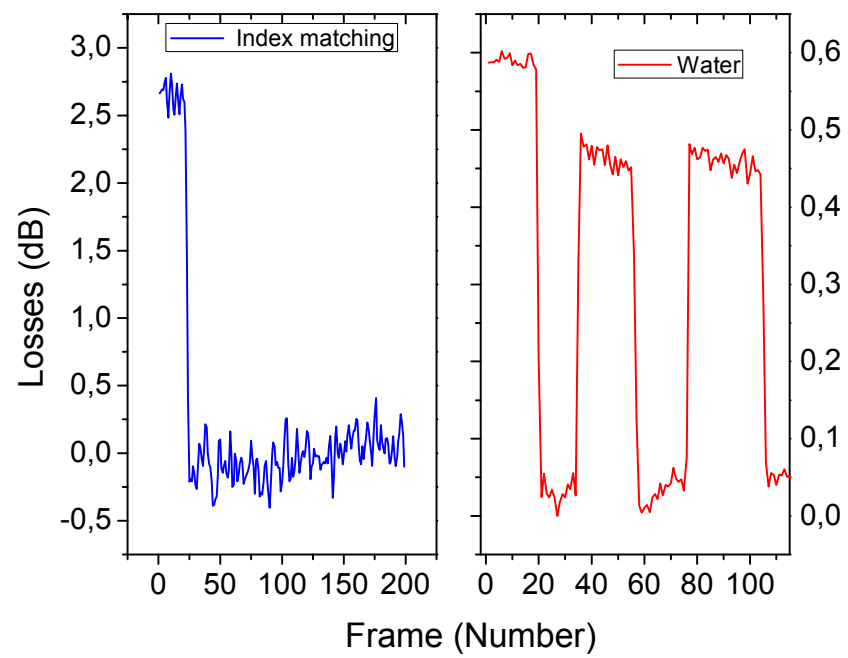

Figure 6. Normalized losses of a speckles pattern section (1x1024) calculated from the THSP matrix in Figure 3.

\section{CONCLUSION}

The capability to detect liquid variation by using the speckles pattern has been shown. The intensity variation, due to the liquid around the sensor, cannot be measured accurately with a typical optical power meter; however it is possible detecting these conditions by analyzing the intensity of the captured image of the speckles pattern.

\section{ACKNOWLEDGMENTS}

The authors acknowledge the Spanish government for supporting this work through the TEC2010-20224-CO2-02 projects and with the grant AP2009-1403.

\section{REFERENCES}

[1] Sirohi, R.S., Ed. Selected Paper on Speckle Metrology, SPIE Milestone Series, MS 35, Washington, USA (1991).

[2] Spillman, W.B.Jr., Kline, B.R., Maurice, L.B., and Fuhr, P.L., "Statistical-mode sensor for fiber optic vibration sensing uses", Applied Optics, 28, 3166-3176, (1989).

[3] Yu, F.T.S., Jing, S., Zhang, J., Guo, R., "Application of a fiber-speckle hologram to fiber sensing", Applied Optics, 33, 502-503, (1994).

[4] Zhang, Z., and Ansari, F., "Fiber-optic laser speckle-intensity crack sensor for embedment in concrete", Sensors and Actuators A-126, 107-111, (2006).

[5] Regez, B., Sayeh, M., Mahajan, A., Figueroa, F., "A novel fiber optics based method to measure very low strains in large scale infrastructures", Measurement, 42, 183-188.

[6] Wang, B., Huang, C., Guo, R., Yu, F.T.S., "A Novel Fiber Chemical Sensor Using Inner-Product Multimode Fiber Speckle Fields”, SPIE vol. 5206, 299-304 (2003).

[7] Goodman, J.W., Speckle Phenomena in Optics, Roberts and Company, Chapter 7, Englewood, Colorado, (2007).

[8] Gloge, D., "Optical-Fiber Packaging and Influence on Fiber Straightness and Loss", B.T.S.J., 54, 245-262 (1975).

[9] G. Jiang, G., Shi, R.F., and Garito, A.F., "Mode Coupling and Equilibrium Mode Distribution Conditions in Plastic Optical Fibers", IEEE Photon. Technol. Lett., 9, 1128-1130 (1997).

[10] Marcuse, D., "Curvature loss formula for optical fibers", J. Opt. Soc. Am., 66, 216-220, (1976).

[11] Donlagic, D., Culshaw, B., "Propagation of the Fundamental Mode in Curved Graded Index Multimode Fiber and Its Application in Sensor Systems", J. Lightwave Technol., 18, 334-342, (2000). 